\title{
The Analytical Method to Compute the Strain on the Soft PSD in Double-Pulse SRM
}

\author{
Chunguang Wang $\mathbb{D}^{1}$ and Guiyang $\mathrm{Xu} \mathbb{D}^{2}$ \\ ${ }^{1}$ College of Electrical and Control Engineering, Shaanxi University of Science \& Technology, Xi'an 710021, China \\ ${ }^{2} X i$ 'an Modern Chemistry Research Institute, Xi'an 710065, China \\ Correspondence should be addressed to Chunguang Wang; king19850723@163.com
}

Received 4 August 2019; Revised 26 September 2019; Accepted 1 October 2019; Published 4 December 2019

Academic Editor: Angelo Cervone

Copyright (C) 2019 Chunguang Wang and Guiyang Xu. This is an open access article distributed under the Creative Commons Attribution License, which permits unrestricted use, distribution, and reproduction in any medium, provided the original work is properly cited.

\begin{abstract}
In order to obtain the analytical method to compute the circumferential strain on a soft pulse separation device (PSD), deformation processes of the middle section of the soft PSD, the medicine propellant grain and the case are simplified into a two-dimensional plane strain state. It is found that the main factors affecting the circumferential strain of the soft PSD are the circumferential strain of the inner surface of the propellant grain and the gap between the soft PSD and the propellant grain. In order to study the failure mechanism of the soft PSD in the double-pulse solid rocket motor (SRM), a two-dimensional axisymmetric finite element method (FEM) model of the stress process of the soft PSD is established. The variation of the strain of the soft PSD with the internal pressure load is obtained. It is found that the excessive circumferential strain is the main reason for the failure of the soft PSD. Comparing the analytical calculations with the FEM results, it can be found that the analytical method value is slightly higher than the FEM value, so the analytical method results can be used to initially estimate the circumferential strain of the soft PSD and then predict the rationality and feasibility of the design scheme. In order to further study the failure mechanism of the soft PSD, a micro-CT test of in situ stretching of the soft PSD material is carried out and the variation of porosity and elongation of the material is studied. The test results showed that when the material elongation is large, the microinterface debonding rapidly expands into a penetrating damage, and the PSD structure fails. The conclusions obtained in this paper can provide a useful reference for the design of double-pulse SRM.
\end{abstract}

\section{Introduction}

A pulse solid rocket motor (SRM) uses the pulse separation device (PSD) to divide the combustion chamber of SRM into several parts to distribute the thrust and the pulse interval time reasonably and improve the performance of various types of tactical missile systems comprehensively. PSD is a difficult and key technology for the development of a dual SRM [1].

In reference [2], the calculation method of the stress intensity factor of the prefabricated defects of the metal diaphragm is obtained by the circular plate large deflection theory and the fracture mechanics theory, and the design basis of the metal diaphragm type compartment in the double SRM is obtained. According to the bearing capacity, PSD can be divided into two typical pulse separation devices: hard PSD and soft PSD. Literature [3-6] studied the char- acteristics of a double-pulse SRM internal flow field. During the second-pulse operation, a backward-facing step flow occurred in the first-pulse combustion chamber due to the contraction of the interstage pulse channel. Thereby, a gas vortex is generated, which exacerbates the convective heat transfer of the first pulse and the particle flushing. In reference [7], the characteristics of the internal flow field of an inner bore clapboard pulse SRM on the condition of pure gas phase and gas-particle phase is studied. In reference [8], the second-pulse ignition transient process of a double-pulse SRM is studied by the self-developed multiphysics coupled solver. In reference [9], the material EPDM is used as a soft PSD for a double-pulse SRM. According to the theory of continuous medium mechanics of rubber materials, a viscous superelastic constitutive model describing the EPDM soft PSD under finite deformation is established. In reference 
[10], the pressurization process of PSD was calculated by FEM and analyzed during the operation of the first grain. Some basic reasons for the damage of PSD during compression were analyzed.

In SRM work, a PSD has a high-pressure, high-temperature, high-stress, and large deformation working environment. In particular, a soft PSD has a large deformation under hightemperature and high-pressure gas. However, a soft PSD is far from reaching the material's elongation, and it is ineffective. The high-temperature gas ignites the second-pulsed propellant grain through the PSD, and the two stages of pulses work simultaneously. The pressure inside the combustion chamber rises rapidly, causing SRM to explode. The structural failure of the soft PSD has become an important issue hindering the development of double-pulse SRM [11].

In this paper, the research work on the failure of a soft PSD in the working process of a double-pulse SRM is carried out. In order to obtain the analytical method to compute the circumferential strain of the soft PSD, the deformation process of the intermediate section of the PSD, the propellant grain and the case are simplified into a two-dimensional plane strain state. This analytical method can be used as a formula basis for the design of a double-pulse SRM. The two-dimensional axisymmetric FEM model of the stress process of the soft PSD is established, and the variation of the strain of the soft PSD with the internal pressure load is obtained to verify the accuracy of the analytic method. In order to further study the failure mechanism of the soft PSD, the micro-CT test of in situ stretching of the soft PSD material is carried out, and the variation of porosity and elongation of the material is studied.

\section{System Description and Critical Failure Mechanism of Soft PSD}

2.1. System Description of PSD. The structure of the soft PSD double-pulse SRM is shown in Figure 1. It includes a first pulse, a second pulse, a soft PSD, and a weak area. The working principle of the soft PSD is shown in Figure 2. During the first-pulse SRM operation, the soft PSD is pressure-bearing through the second-pulsed propellant grain. At the same time, it acts as a heat insulator and seal. The reliability of the seal and pressure is directly related to the safety of the second grain. As a result of the pressure from the first grain, the soft PSD will produce radial deformation with the second grain, resulting in circumferential strain. When the secondpulse grain is working, the soft PSD first breaks in the weak area. Then, the broken section is reversed and turned open. Therefore, a reasonable design not only requires the second pulse to work reliably at a predetermined pressure and a predetermined weak area but also requires the weak area section to be smoothly reversed and turned open, thereby ensuring reliable ignition and a stable combustion flow of the second-pulse grain [10].

The thickness of soft PSD is generally about $8 \mathrm{~mm}$. There is a mold release agent between the soft PSD and the second grain to ensure that the grain and soft PSD are separated. In addition, a natural gap will be formed between the soft PSD and the grain due to radial contraction during the curing

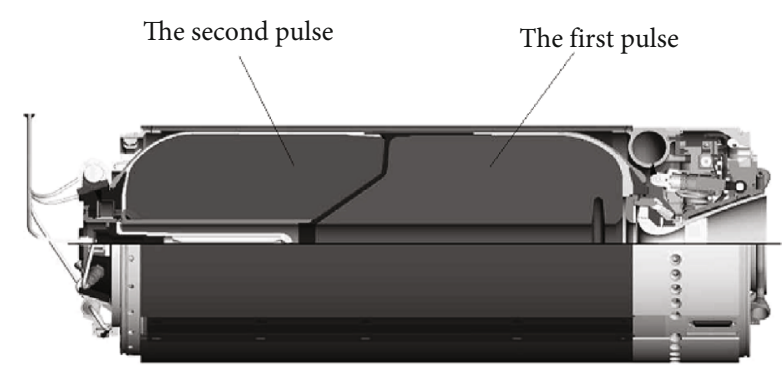

Figure 1: Soft PSD double-pulse SRM.

and cooling process of the propellant. Due to the fluctuation of the processing process, the gap is generally between 1 and $5 \mathrm{~mm}$.

2.2. Critical Failure Mechanism of Soft PSD. Through the cold gas test and the single test of the first pulse, it is found that the failure of PSD is generally caused by excessive circumferential strain. Thereby, it is very important to obtain the calculation method to compute the strain for PSD. Since SRM has a certain length, the strain can be approximated as a plane strain problem at the middle section of the soft PSD.

The calculation of the circumferential strain in the middle of the soft PSD can be simplified to formula (1). $\varepsilon_{\theta}$ is the circumferential strain of the soft PSD. $a$ is the inner diameter of the propellant grain, $a^{*}=a-\delta_{0}$ is the diameter of the soft PSD, and $\delta_{0}$ is the gap between the soft PSD and the propellant grain; the simplified form is shown in Figure 3.

$$
\varepsilon_{\theta}=\frac{\Delta R}{a-\delta_{0}-\delta_{g}}
$$

Among them, $\Delta R$ contains two parts: $\delta_{0}$ and $\delta_{g} . \delta_{g}$ is the radial displacement generated by the surface of the propellant grain under internal pressure.

$\delta_{g}$, which is the radial displacement of the inner surface of the propellant grain, can be obtained by the calculation formula (2).

The specific derivation process can be found in the literature $[12,13]$.

$\delta_{g}=\frac{1+v}{\left(m^{2}-1\right) E}\left\{[(1-2 v) a+m R] p-\left[m R+(1-2 v) m^{2} a\right] q\right\}$,

where $m$ is the ratio of the outer diameter to the inner diameter of the propellant grain, $p$ is the internal pressure load of the SRM generated by first grain burning, $v$ is the Poisson's ratio of the propellant grain, $E$ is the elastic modulus of the propellant grain, and $q$ is the external pressure generated by the constraint of the case. The calculation method is shown as follows $[12,13]$ :

$$
q=\frac{4\left(1-v^{2}\right) P}{2(1+v)\left[1+(1-2 v) m^{2}\right]+\left(2-v_{k}\right)\left(m^{2}-1\right)\left(E R / E_{k} h\right)},
$$




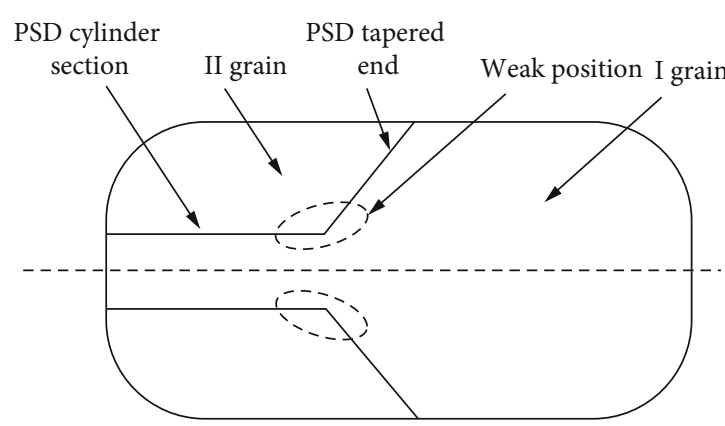

FIgURE 2: Working principle of PSD.

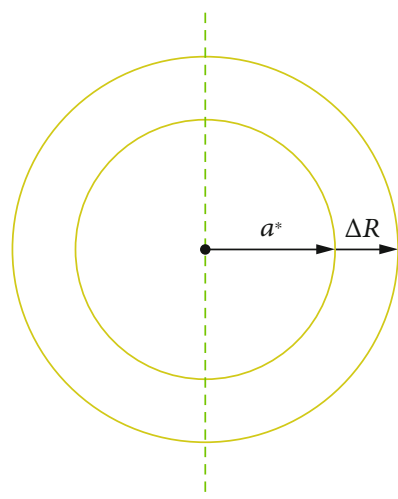

FIGURE 3: Plane strain.

where $v_{k}$ is the Poisson's ratio of the case and $E_{k}$ is the modulus of elasticity of the case. If the case material is composite, then $v_{k}$ is the ring longitudinal Poisson's ratio and $E_{k}$ is the circumferential modulus.

Formulas (2) and (3) are brought into formula (1) to obtain the approximate calculation formula of the circumferential strain of the soft PSD under the action of internal pressure load, as shown in formula (4):

$$
\begin{aligned}
\varepsilon_{\theta} & =\frac{\delta_{0}}{a}+\varepsilon_{\theta g}, \\
\varepsilon_{\theta g} & =\frac{1+v}{\left(m^{2}-1\right) E}\left\{\left[(1-2 v)+m^{2}\right] p-\left[m^{2}+(1-2 v) m^{2}\right] q\right\} .
\end{aligned}
$$

It can be seen from formula (4) that for the double-pulse SRM, which has determined the geometry of the propellant type, the main factors affecting the circumferential strain of the soft PSD are the circumferential strain of the propellant grain inner surface and the gap between the soft PSD and the propellant grain.

\section{Compartment Stress Analysis}

3.1. Calculation Model. In order to verify the accuracy of the analytic method, FEM calculation is carried out. According to the stress state of the soft PSD, the structure of the double-pulse SRM is simplified, and a two-dimensional axisymmetric model is established to analyse the structural

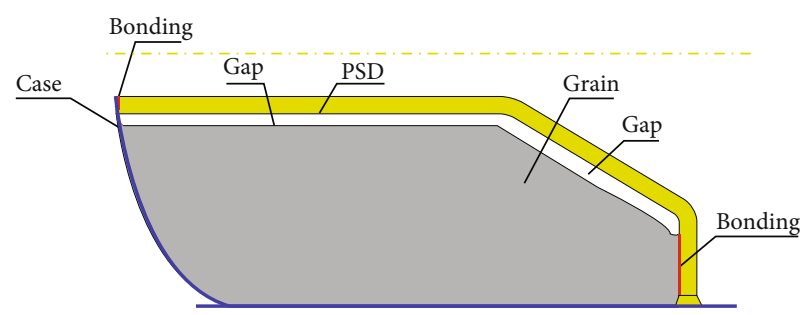

Figure 4: Simplified physical model of second-pulse combustion chamber.

response characteristics of the double-pulse SRM under first-pulse grain burning. As shown in Figure 4, the SRM is composed of a metal case, a second-pulsed propellant grain, and a soft PSD. The gap between the propellant grain and the PSD is valued according to the process detection data.

3.2. Calculation Method. The following assumptions are made regarding the operating characteristics of the SRM:

(a) The useful temperature range for the EPDM material is about $-55^{\circ} \mathrm{C} \sim+150^{\circ} \mathrm{C}$. When first-pulse grain is burning, high-temperature combustion gas is stationary on the surface of the soft PSD and the heat transfer speed to the soft PSD is not fast. The temperature of the surface of the soft PSD will rise by $80^{\circ} \mathrm{C}$, and its overall average temperature will rise by $30^{\circ} \mathrm{C}$, hardly affecting its mechanical properties. According to the experimental results, the thermal load is not the cause of failure of the soft PSD. As a result, the temperature rise and ablation of the soft PSD are negligible, and the performance change of the PSD material caused by the temperature change is not considered

(b) Quasi-static analysis is performed for a given load without considering the inertial effect of structural mass. The effect of strain rate on the mechanical properties of EPDM and propellant grain is not considered too

(c) The friction between the soft PSD and the structural parts, such as the case and the second-pulsed propellant grain, is ignored

(d) The pressure of the first-pulse grain burning pressurization is evenly applied to the soft PSD

The internal pressure load is taken as the maximum working pressure of the SRM, which reaches to $9.0 \mathrm{MPa}$, and a fixed constraint is applied to the front skirt of case end face to eliminate the rigid body displacement, as shown in Figure 5.

In order to simulate the pressure building process of the SRM internal pressure, the pressure load curve is loaded as shown by the curve change trend in Figure 6 .

3.3. Material Properties. According to engineering development experience, the internal pressure of the SRM is usually established within $0.3 \mathrm{~s}$, and the propellant grain and thermal 


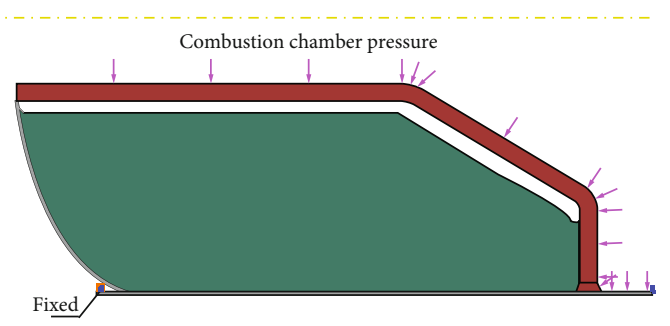

FIGURE 5: Second-pulsed propellant grain stress.

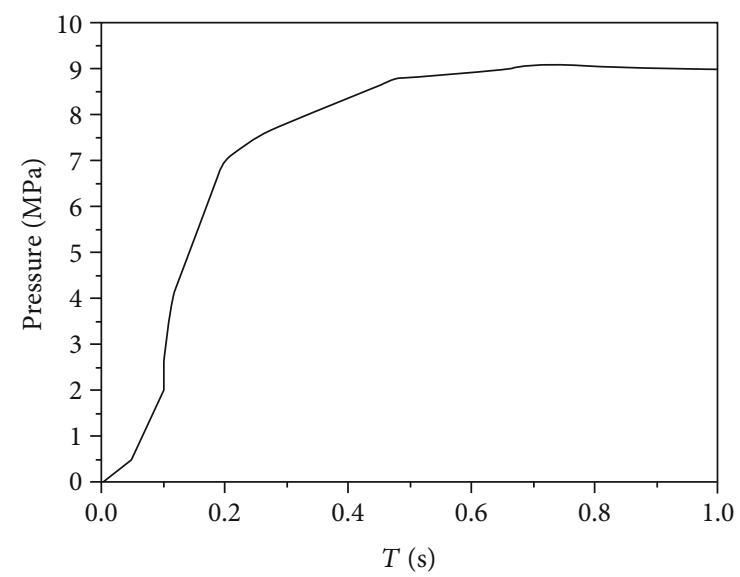

Figure 6: Internal pressure curve of SRM.

TABle 1: Performance parameters of the SRM.

\begin{tabular}{lcc}
\hline Structure & Modulus & Poisson's ratio \\
\hline Case & $200 \mathrm{GPa}$ & 0.3 \\
Second-pulsed propellant grain & $5.2 \mathrm{MPa}$ & 0.495 \\
Soft PSD & $10.0 \mathrm{MPa}$ & 0.495 \\
\hline
\end{tabular}

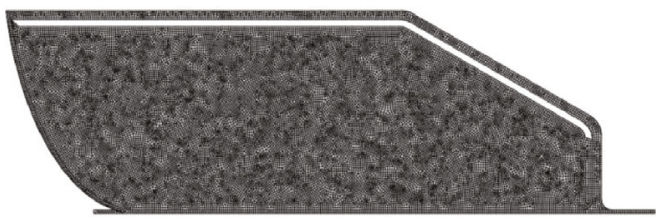

Figure 7: Model overall grid.

insulation layer (including the compartment) can be simulated as a linear elastic material. The performance parameters of each material of the SRM are shown in Table 1.

3.4. Computing Grid. The geometric model is meshed under ABAQUS/CAE for a total of 60,000 finite element meshes, as shown in Figure 7. For volume approximate incompressible material characteristics of the propellant grain and short fiber reinforced EPDM, the hybridization unit option is selected in the unit setting under ABAQUS/CAE. And the Plane Axisymmetric Hybridization unit (CAX4H) is selected to avoid the volume self-locking and finite element analysis by the high Poisson ratio, which is closest to 0.5. Linear Reduced Integral Planar Axisymmetric Unit (CAX4R) is used for the case. Figure 8 is a partial magnification of the mesh.

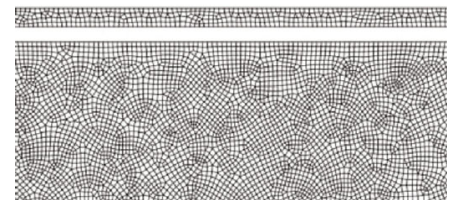

Figure 8: Local grid.

The interface of the case and grain is the bonding condition. The interface of PSD and the grain is the contact in the gap condition. The interface of PSD and the case is the bonding condition.

3.5. Result Analysis. After the calculation is completed, the strain in the three directions of the soft PSD is shown in Figure 9.

According to the calculation results, it can be found that

(1) The radial strain in the middle of the soft PSD is negative, meaning that the central portion is radially compressed. And there is a high tensile strain zone at both ends due to adhesion to the case, but the area is extremely small

(2) The two segments of the PSD are bound to the case and end face of the grain. The interface of the case and grain is the bonding condition. The axial deformation of the propellant/case combination structure causes the axial strain of the soft PSD. It is worth noting that the main axial strain still occurs at the two ends of the soft PSD bound to the case, and most of the soft PSD has a small axial strain, especially the middle section

(3) The main strain of the soft PSD is the circumferential strain. The largest part of the circumferential strain is in the axial middle section of the soft partition, reaching a maximum of $21.05 \%$

The maximum strain point of the high-strain region of the soft PSD is recorded as point A, as shown in Figure 10.

Circumferential strain versus time curves of point $A$, the propellant grain inner surface, and the case of the same axial position as point A are shown in Figure 11.

It can be seen from Figure 10 that when the first-pulse grain working pressure acts on the soft PSD, the trend of the circumferential strain of the PSD, the propellant grain, and the case are similar to the loading trend of the internal pressure. The development of the circumferential strain can be divided into the following three stages:

(1) The first stage: the gap between the soft PSD and the propellant grain is quickly filled, and the circumferential strain of the soft PSD is mainly due to the filling gap, and its value accounts for $26.5 \%$ of the total strain of the whole process

(2) The second stage: the propellant grain and the soft PSD as a whole limit radial displacement of the soft PSD after the soft PSD is in contact with the propellant grain. Under further action of the pressure, the 


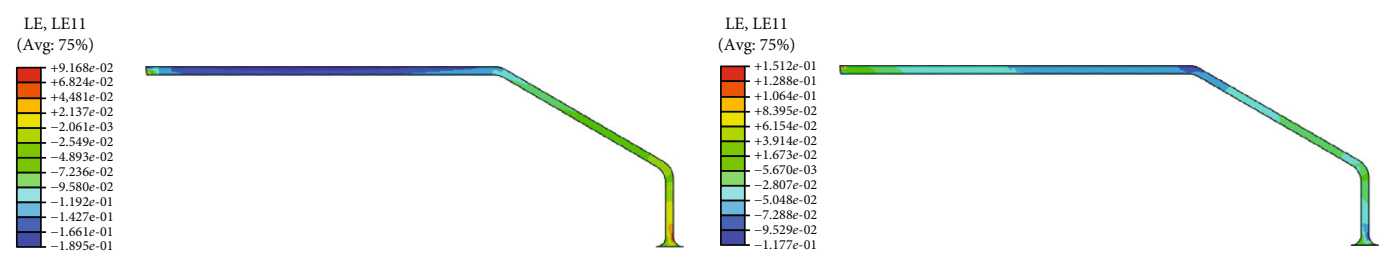

(a) Radial strain

(b) Axial strain

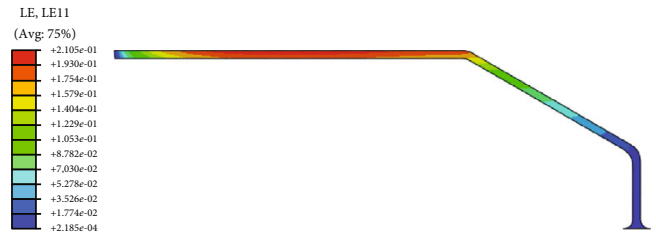

(c) Circumferential strain

FIGURE 9: PSD strain contours.

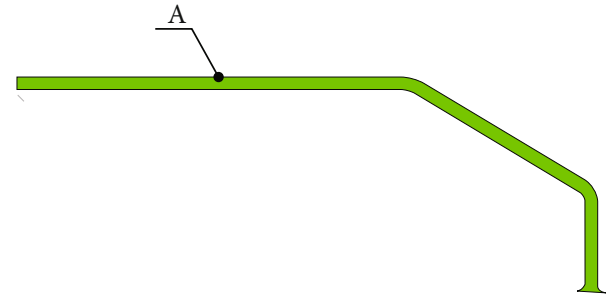

FIgURE 10: The maximum strain point of the soft PSD (point A).

whole grain and the case are deformed under pressure, and the circumferential strain is further increased

(3) The third stage: due to the approximate incompressibility of the propellant grain, the radial deformation of the grain is getting smaller and smaller, and the circumferential strain gradually approaches the maximum value under the limitation of the case

\section{Analysis of Strain Sensitivity Factors}

In order to verify the accuracy of the analytical method results, several FEM calculations were carried out, based on the basic assumptions, loads, and boundaries described above.

The strain results of the soft PSD and the inner hole of the propellant grain are shown in Table 2, under a different elastic modulus of the case and the gaps between the grain and soft PSD. It is worth noting that when calculating the $m$ of the propellant grain, it is necessary to consider the influence of the deformation of the propellant grain due to the internal pressure. The propellant grain inner diameter after the deformation is equal to the design value of the inner diameter plus the deformation of the propellant grain inner diameter obtained by the FEM calculation. The specific calculation formula is shown in formula (6), where $\delta_{\mathrm{FEA}}$ is the increase in the propellant grain inner diameter.

$$
m=\frac{R}{a+\delta_{\mathrm{FEA}}} .
$$

It can be found from Table 2 that when the gap increases, the circumferential strain of the soft PSD increases, and when the stiffness of the case increases, the circumferential strain of the soft PSD decreases. And the effect of the gap between the soft PSD and the propellant grain on the circumferential strain of the soft PSD is obvious.

In order to verify the accuracy of the analytical method, another new example is carried out. The results of the calculation and comparison are shown in Table 3.

Comparing the analytical method results with the FEM results, we can find that the results are basically the same, and the analytical values are slightly higher than the FEM values. This is because the propellant grain section strain is treated as a plane strain, ignoring the axial strain caused by the compression of the propellant grain, resulting in a small calculated value of the axial stress, and according to the three-dimensional stress-strain relationship in the elastic mechanics, the calculated value of the axial stress is too small, resulting in a large calculation of the circumferential strain, during the derivation of formula (4).

Considering the strain of the soft PSD alone, the calculated value of the formula is about $10 \%$ higher than the FEM value, which is more conservative. Therefore, the strain of the soft PSD can be estimated by formula (4) to determine the rationality and feasibility of the design in the engineering design. For further in-depth evaluations, further FEM is used for more detailed calculations.

\section{Experimental Study on Failure Mechanism of Rubber Materials for Soft PSD}

The soft PSD is generally made of special rubber, and it is mainly composed of two parts: a matrix EPDM and a chopped fiber filler. The rubber material not only has hightemperature resistance, low density, and high elongation but it also has a certain structural strength. As mentioned above, the soft PSD is deformed under the action of hightemperature and high-pressure gas; however, it is far from reaching the material's elongation, and the soft PSD is ineffective, causing the two-stage pulse to work at the same time, and the case is detonated and exploded [14]. 


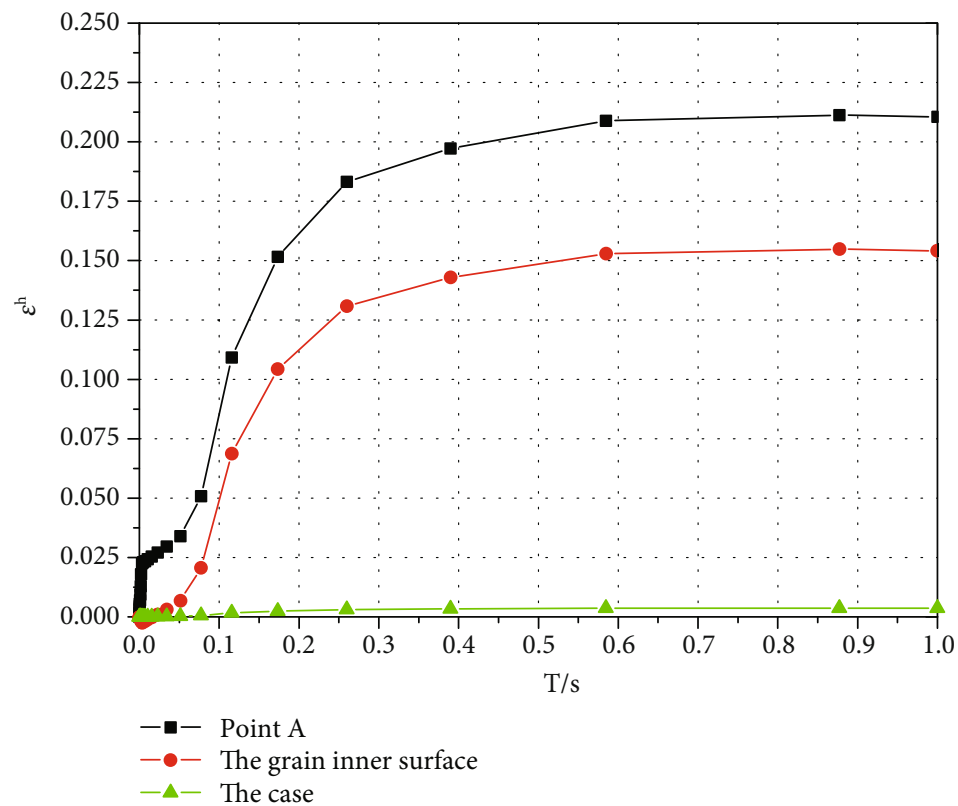

FIgURE 11: Circumferential strain versus time curve of point A, the propellant grain inner surface, and the case.

TABLE 2: Calculation results of circumferential strain.

\begin{tabular}{ccccccc}
\hline & \multicolumn{2}{c}{ Input } & \multicolumn{4}{c}{ Structural response } \\
& Gap/mm & $\begin{array}{c}\text { Case } \\
\text { modulus } \\
(\mathrm{GPa})\end{array}$ & $\begin{array}{c}\varepsilon_{\theta} \text { of case } \\
\text { FEM }\end{array}$ & $\begin{array}{c}\text { Analytical } \\
(\%)\end{array}$ & $\begin{array}{c}\text { FEM } \\
\varepsilon_{\theta} \text { of grain }\end{array}$ & $\begin{array}{c}\text { Analytical } \\
(\%)\end{array}$ \\
\hline 1 & 1.5 & 150 & 21.67 & 23.71 & 15.99 & 21.29 \\
2 & 5.0 & 150 & 25.85 & 28.29 & 14.89 & 19.74 \\
3 & 1.5 & 200 & 21.05 & 23.21 & 15.46 & 20.79 \\
4 & 5.0 & 200 & 25.26 & 27.81 & 14.39 & 19.27 \\
5 & 1.5 & 220 & 20.82 & 23.03 & 15.27 & 20.61 \\
6 & 5.0 & 220 & 25.05 & 27.64 & 14.21 & 19.09 \\
\hline
\end{tabular}

Note: the circumferential strain of the soft PSD takes the maximum value at the end of the loading, and the circumferential strain of the case takes at the same axial position as the maximum circumferential strain of the soft PSD.

In order to further explore the failure mechanism of the soft PSD material, a micro-CT test of in situ stretching is carried out to study the correlation between the mesodamage degree of the soft PSD material and the strain. The change in the meso-level of the test piece is characterized by porosity. The reconstruction and variation of the two-dimensional porosity image is shown in Figure 12.

It can be found from Figure 12 that when the elongation is between 0 and $10 \%$, the porosity remains substantially unchanged, indicating that no debonding occurs between the fibers and the filler; when the elongation is between $10 \%$ and $30 \%$, the porosity increases linearly, and the fiber and the solid filler have a small amount of debonding with the matrix; when the elongation is greater than $30 \%$, the porosity increases rapidly; when the elongation is greater than $50 \%$, the debonding region has already produced a relatively large and through damage.

The main reasons why the soft PSD fails at lower elongation are as follows: when the circumferential strain of the soft
TABLE 3: Calculation results of circumferential strain of another example (a double-pulse SRM with twice the diameter; $R / h$ stays the same).

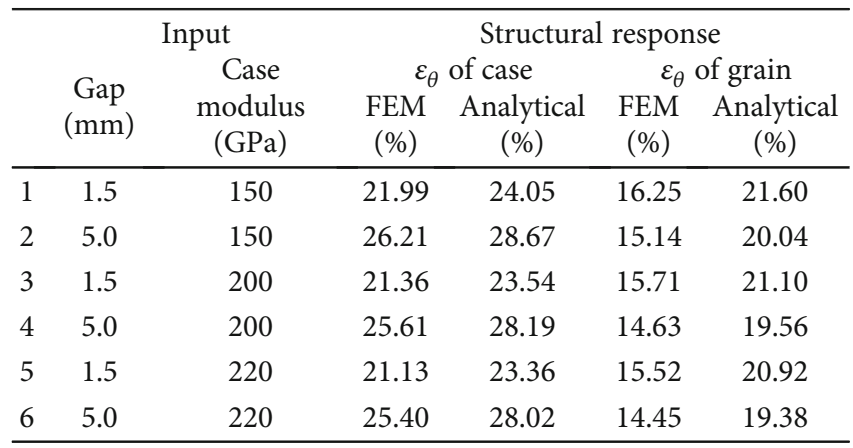

PSD material is increased to a certain extent, debonding between the chopped fibers and the rubber matrix begins to occur. As the strain continues to increase, the fiber is peeled off and the porosity increases until the through damage occurs, and the soft PSD fails.

\section{Conclusions}

(1) The soft PSD strain is mainly caused by circumferential deformation by the load of the first-pulse grain internal pressure. The largest part of the circumferential strain is in the axial middle section of the soft PSD. It is considered that the circumferential strain is the main cause of the failure of the soft PSD

(2) The circumferential strain of the soft PSD is related to the gap between the soft PSD and the propellant grain and the stiffness of the case. In the SRM manufacturing process, the shrinkage deformation of the soft PSD material and the propellant grain 


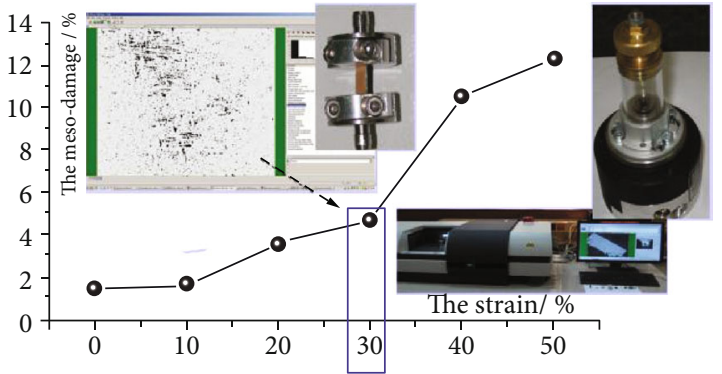

FIGURE 12: Porosity of soft PSD material varies with elongation.

should be considered, and the gap between the propellant grain and the soft PSD should be as small as possible after the propellant grain is cured. In addition, under the same conditions, if the case has high stiffness, it is advantageous for the integrity of the PSD

(3) In this paper, the deformation process of the soft PSD is simplified to the two-dimensional plane strain problem, and the calculation method to compute the internal surface strain of the soft PSD is obtained. The calculation results of this method are in good agreement with the FEM results and can be used for the strain estimation of the soft PSD to preliminarily determine the rationality and feasibility of the design scheme

(4) In situ tensile/micro-CT scanning experiments are carried out on the soft PSD material to study the variation of porosity and elongation. When the material elongation is large, the micro-interface debonding rapidly expands into a penetrating damage, and the soft PSD structure fails. This phenomenon can better explain why the SRM disintegrates when the soft PSD material is far below the elongation at break, under the first-pulse grain operation

\section{Data Availability}

As most of the data in this manuscript were related to trade secrets, I cannot provide them completely. In the future, if necessary, I can share some data with reviewers or readers.

\section{Conflicts of Interest}

The authors declare that there is no conflict of interest regarding the publication of this paper.

\section{Acknowledgments}

This article was funded by Xi'an Modern Chemistry Research Institute, China.

\section{References}

[1] C. G. Wang, H. C. Liu, D. M. Yang, and C. Chen, "Study of development status and future development proposals for pulse soild rocket motor," Applied Mechanics and Materials, vol. 110-116, pp. 2354-2358, 2011.
[2] W. K. Liu and B. Hui, "Dynamic and static opening of metal diaphragm in double pulse solid rocket motor," Journal of Propulsion Technology, vol. 35, no. 9, pp. 1259-1264, 2014.

[3] A. Javed, P. Manna, and D. Chakraborty, "Numerical simulation of a dual pulse solid rocket motor flow field," Defence Science Journal, vol. 62, no. 6, pp. 369-374, 2012.

[4] A. Javed and D. Chakraborty, "Universal erosive burning model performance for solid rocket motor internal ballistics," Aerospace Science and Technology, vol. 45, pp. 150-153, 2015.

[5] K. W. Naumann, L. Stadler, P. Trouillot, A. Weigand, D. Zanelli, and S. Schilling, "Double-pulse solid rocket technology at Bay-ern-Chemie/Protac," in 42nd AIAA/ASME/SAE/ASEE Joint Propulsion Conference and Exhibit, Sacramento, California, July 2006AIAA 2006-4761.

[6] W. B. Zhu, Y. F. Zhang, H. Chen, and R. P. Sun, "The study of dual-pulse solid rocket motor's inner flow field," Journal of projectiles, Rockets, Missiles and Guidance, vol. 32, no. 1, pp. 114-118, 2012.

[7] X. W. Cao, Y. Liu, and X. J. Ren, "Analysis on flow field of inner bore clapboard pulse solid rocket motor," Journal of Aerospace Power, vol. 26, no. 2, pp. 448-452, 2011.

[8] Y. K. Li, J. L. Han, and X. Chen, "Numerical simulation of the ignition transient of dual pulse motor based on multi-physics coupling," Acta Aeronautica et Astronautica Sinica, vol. 38, no. 4, pp. 80-91, 2017.

[9] X. G. Fang, J. S. Xu, and X. Chen, "A visco-hyperelastic constitutive model for EPDM soft PSD of dual-pulse motors," Acta Aeronautica et Astronautica Sinica, vol. 39, no. 11, pp. 115123, 2018

[10] P. Fu, X. Y. Song, L. Q. Sun, and D. Yao, "Investigation on the reverse opening process of the soft insulator of double pulse solid rocket motor," Journal of Solid Rocket Technology, vol. 40, no. 2, pp. 146-151, 2017.

[11] C. G. Wang, W. P. Tian, C. Chen, and D. M. Yang, "Investigation on the work process of soft PSD in pulse SRM," Missiles and Space Vehicles, vol. 324, no. 1, pp. 11-16, 2013.

[12] R. X. Chen and Y. Chen, "Relationship between grain inner surface train and case strain," Journal of Solid Rocket Technology, vol. 36, no. 6, pp. 763-765, 2013.

[13] R. X. Chen, "Effect of case stiffness on the grain strength when applied internal pressure," Journal of Solid Rocket Technology, vol. 34, no. 1, pp. 101-104, 2011.

[14] S. S. Liu, X. Hou, and J. Gao, "Strength and invalidation analysis of elastomeric barrier reinforced by short-staple fiber," Journal of Solid Rocket Technology, vol. 40, no. 1, pp. 100104, 2017. 


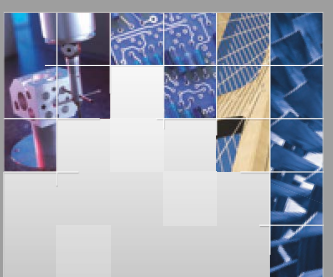

\section{Enfincering}
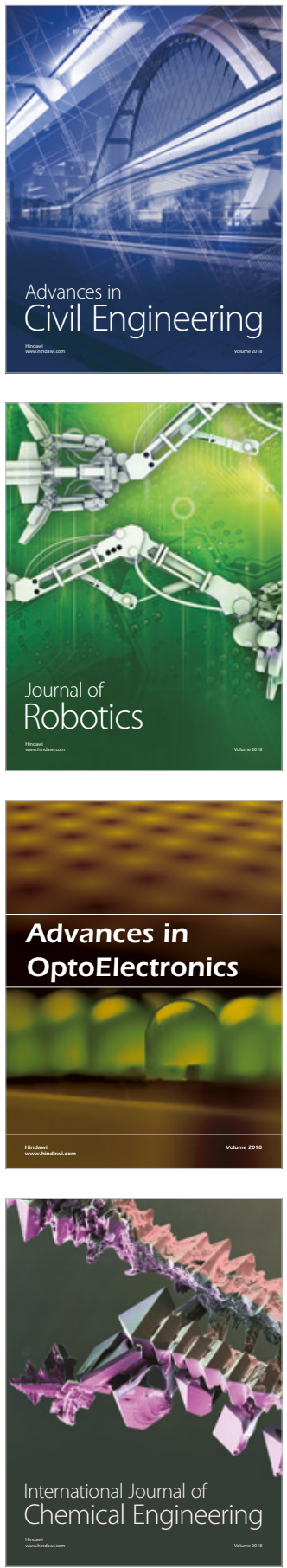

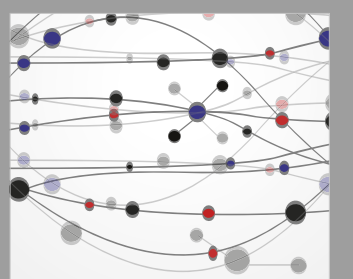

\section{Rotating \\ Machinery}

The Scientific World Journal

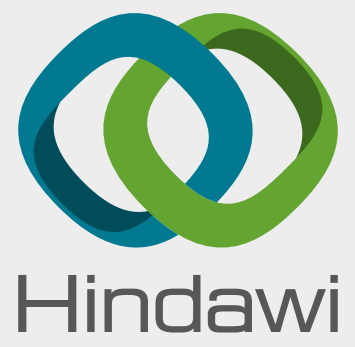

Submit your manuscripts at

www.hindawi.com
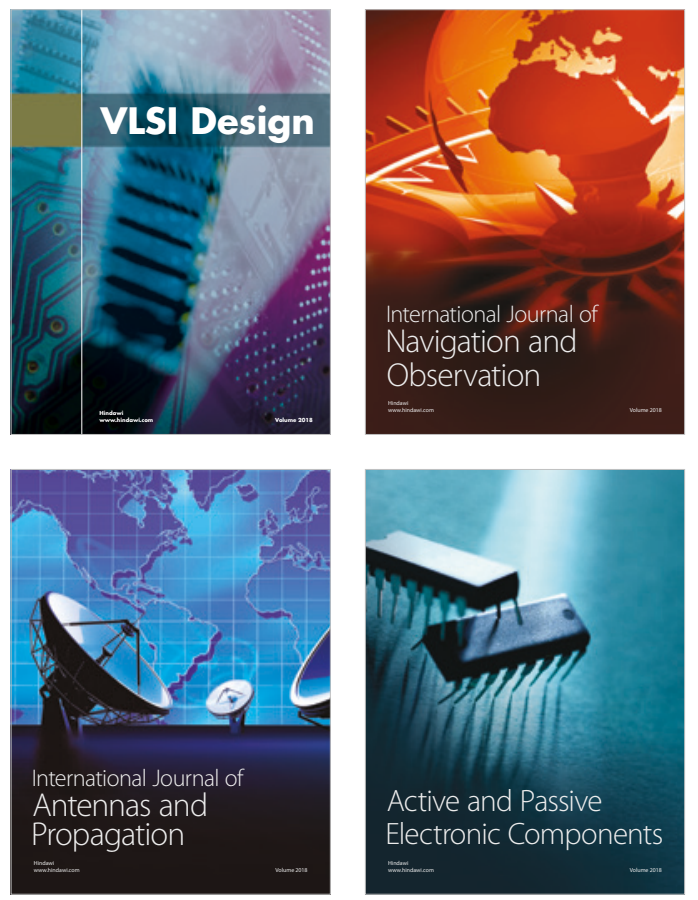
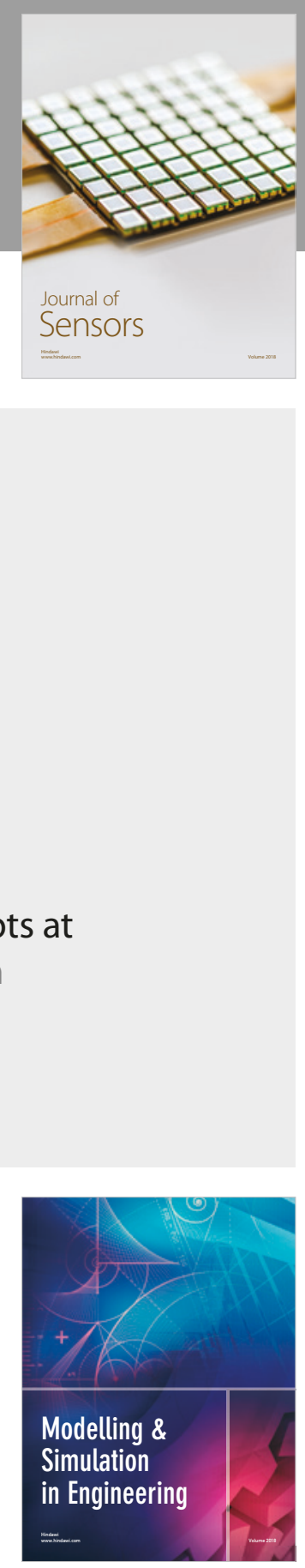

\section{Advances \\ Multimedia}
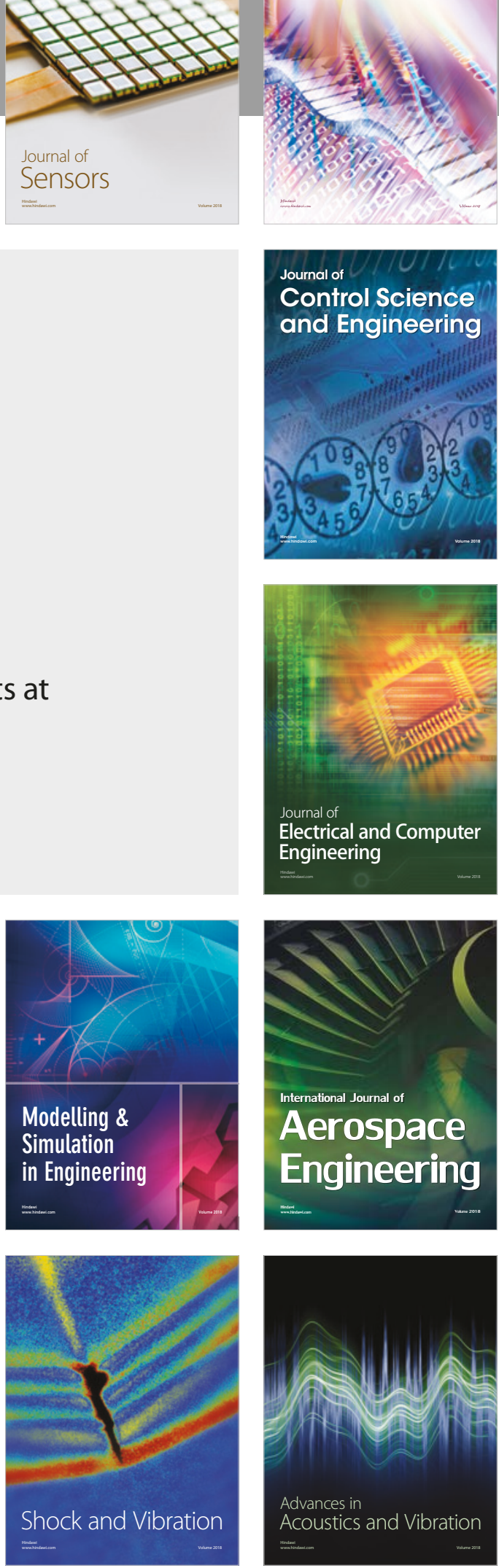